\title{
Quench dynamics of dipolar fermions in a one-dimensional harmonic trap
}

\author{
Tobias Graß \\ ICFO (Institut de Ciències Fotòniques), Avinguda Carl Friedrich Gauss 3, 08860 Castelldefels (Barcelona), Spain
}

(Received 23 July 2015; published 25 August 2015)

\begin{abstract}
We study a system of few fermions in a one-dimensional harmonic trap and focus on the case of dipolar majority particles in contact with a single impurity. The impurity is used both for quenching the system and for tracking the system evolution after the quench. Employing exact diagonalization, we investigate relaxation and thermalization properties. In the absence of dipolar interactions, the system dynamics remains oscillatory even on long time scales. On the other hand, repulsive as well as attractive dipolar interactions lead to quick relaxation to the diagonal ensemble average, which is significantly different from corresponding thermal averages. A Wigner-shaped level spacing distribution indicates level repulsion and thus chaotic dynamical behavior due to the presence of dipolar interactions.
\end{abstract}

DOI: 10.1103/PhysRevA.92.023634

PACS number(s): $03.75 . S s, 34.50 . C x$

\section{INTRODUCTION}

From the microscopic point, of view physical systems are governed by the laws of quantum mechanics. For systems of macroscopic size, however, a description on this level usually fails due to the huge amount of different degrees of freedom. In such case, a tractable approach is to take appropriate averages over the degrees of freedom, replacing the microscopic laws by the laws of statistical mechanics. This switch has some paradoxical consequences, such as the emergence of irreversible dynamics from microscopic laws with time-reversal symmetry. In order to reconcile the physical laws on the microscopic and the macroscopic levels, it seems to be necessary to extend our microscopic understanding towards larger system sizes. Therefore, we need to improve our capabilities in obtaining exact or quasiexact knowledge about a system.

Maybe the most promising direction to achieve this goal is quantum simulations, that is, experiments with wellcontrolled, tunable quantum systems [1]. A remarkable step towards bridging the gap between microscopic and macroscopic physics was the recent experiments in Heidelberg with cold fermionic atoms in a one-dimensional trap [2-4]. Outstanding for the precise control over particle numbers, the experiment of Ref. [4] demonstrated the formation of a so-called Fermi sea of up to five majority atoms interacting with a single impurity atom. These experimental advances have also boosted the theoretical interest in one-dimensionally trapped Fermi [5-18] and Bose [18-25] systems.

In this article, we extend the scenario from the Heidelberg experiments into two directions: First, we assume dipolar interactions between the fermions. Second, we shift the focus onto dynamical processes. The standard interactions in quantum gases are contact interactions. With this, a spin-polarized Fermi sea as in Ref. [4] remains noninteracting, since contact interactions are forbidden by the Pauli principle. Clearly, the most prominent fermions in nature are electrons with longrange Coulomb interactions. To design an atomic quantum simulation of a Fermi systems with long-range repulsion, one might stick to dipolar interactions. The long-range character of these interactions is certainly one of various reasons for the huge research interest in dipolar quantum gases [26-36]. With advances in cooling molecular systems [37,38] and recent developments involving Rydberg gases [39-41], other experimental platforms involving strong dipolar interactions are emerging. One-dimensional systems with dipolar interactions can provide a particularly interesting scenario, as the confinement modifies the effective interaction potential with the possibility of confinement-induced resonances [42-44].

In a system which is strongly confined to one dimension, dipolar interactions become almost local. Therefore, only very strong dipolar interactions significantly modify the ground state of a small Fermi gas. Nevertheless, the dynamical behavior of the system might be affected strongly even by weak interactions. Accordingly, our paper is concerned with the dynamics of the system. Certainly, from the point of view of numerics, dynamics is the most difficult aspect of a quantum system, and therefore best suited to be studied via a quantum simulation. Quantum systems out of equilibrium, and the relation between unitary time evolution and thermodynamics, has attracted broad research interest, cf. Refs. [45-56] and the review articles, Refs. [57,58]. A closely related subject are Lieb-Robinson bounds [59] and the velocity of correlation spreading, in particular in the presence of long-range interactions [60-66]. The scenario which we study here resembles closely the so-called flutter dynamics of an impurity in a homogeneous one-dimensional quantum gas, which has attracted a large amount of attention during the past years [67-73].

In this paper, we study a one-dimensional Fermi system in which majority atoms interact, via contact, with a single impurity, as in the Heidelberg experiments. Among themselves, the majority atoms can interact via dipolar interactions. We first provide a brief description of this system and introduce the effective dipole-dipole interaction in Sec. II. In Sec. III, we sketch the concept of time evolution in quantum mechanics and define some useful quantities to describe the dynamics of a many-body system. After briefly studying static properties of the ground state in Sec. IV, we focus on relaxation processes from an out-of-equilibrium state in Sec. V. Here, the impurity will serve, in a twofold way, as a control knob: On the one hand, we can excite the system by exciting the impurity atom to higher oscillator levels. On the other hand, the impurity can serve as a kind of thermometer. We investigate under which conditions the energy of the impurity atom relaxes to a constant value, and whether this value corresponds to the thermalized value. It will turn out that dipolar interactions 
between the majority atoms significantly enhance relaxation. Generally, the diagonal ensemble describes well the relaxed state, but it differs from thermalized values. Finally, in Sec. VI, we relate our results to more general properties of the system and analyze the level spacing distribution. Although not an integrable model, the system without dipolar interactions exhibits a strong tendency for level clustering. On the other hand, a Wigner-shaped distribution for the system with dipolar interactions indicates level repulsion and chaotic dynamics. In this sense, the level spacing distribution turns out to be a good indicator for the system's relaxation properties. In the appendix, we calculate the interaction matrix elements of a dipolar gas in one dimension.

\section{SYSTEM}

We consider a system of fermionic atoms in an effectively one-dimensional harmonic trap $V_{\text {trap }}=\frac{m}{2} \omega^{2} x^{2}$, where $m$ is the mass of the atoms and $\omega$ is the axial trapping frequency, chosen along the $x$ direction. The one-dimensional trapping is achieved via a sufficiently strong anisotropy of the threedimensional trap, freezing out the dynamics in the transverse directions. The ratio between axial trapping frequency $\omega$ and the transverse trapping frequency $\omega_{\perp}$ is given by the parameter $\lambda=\sqrt{\omega / \omega_{\perp}}=l_{\perp} / l$, with $l=\sqrt{\hbar /(m \omega)}$ and $l_{\perp}=$ $\sqrt{\hbar /\left(m \omega_{\perp}\right)}$ the corresponding harmonic oscillator lengths. For most of our numerical studies, we choose $\lambda=1 / 3$, the value reported in Refs. [2,4]. We work in harmonic oscillator units of the axial trapping, that is, $\omega=1, l=1, m=1$, and $\hbar=1$.

The fermions can be prepared in different internal states, or, equivalently, the system may consist of different species with equal mass. Yet another possibility are identical fermions trapped in two parallel tubes [19]. We restrict ourselves to two-component systems, where $N_{\uparrow}$ and $N_{\downarrow}$ denote the numbers of fermions in each component. We focus on the case where several majority atoms (forming the $\uparrow$ component) interact with a single impurity atom $\left(N_{\downarrow}=1\right)$ via contact interactions, $V_{\text {contact }}=g \sum_{i \in \uparrow, j \in \downarrow} \delta\left(x_{i}-x_{j}\right)$, with effective interaction strength $g$. Such a scenario has been studied experimentally in Ref. [4]. Since identical fermions do not interact with each other via contact interaction, the Fermi sea of the majority systems would be noninteracting. To overcome this, we may equip the majority atoms with an electric or magnetic dipole moment $d$, which leads to dipole-dipole interactions between the majority atoms. For simplicity, we assume that the impurity atom carries no dipole moment, such that it interacts with the majority atoms only via contact interaction.

The dipoles shall be aligned, forming an angle $\theta$ with the $x$ axis. Then, the scattering between dipoles, separated by a vector $\vec{r}$, is described by the usual interaction potential $V_{\mathrm{dd}}(\vec{r})=\frac{d^{2}}{r^{3}}\left(1-3 \cos ^{2} \theta_{r d}\right)$, with $\theta_{r d}$ being the angle between the dipole and $\vec{r}$. Due to the strong transverse confinement, one can assume that transverse degrees of freedom will not be excited, and integrate out the $y$ and $z$ variables. This leads to the effective potential [42-44]

$$
V_{\mathrm{dd}}^{\mathrm{eff}}(x)=-\frac{d^{2}[1+3 \cos (2 \theta)]}{8(\lambda l)^{3}} U(x / \lambda) \equiv u U(x / \lambda),
$$

with the dimensionless potential

$$
U(x)=-2|x|+\sqrt{2 \pi}\left(1+x^{2}\right) e^{x^{2} / 2} \operatorname{erfc}(x / \sqrt{2}),
$$

where erfc is the complementary error function. An additional term $\propto \delta(x)$ occurring in the effective potential can be neglected for identical fermions. We note that the strength of the dipolar interactions, $u$, can be tuned via the polarization angle, $\theta$, and can be made attractive or repulsive.

The total Hamiltonian is studied by exact diagonalization in the Fock basis of the harmonic oscillator levels. We truncate this basis, taking into account up to 22 single-particle levels, for systems of up to five majority atoms. We consider interaction strengths up to $g=10$ and $|u|=10$. In second quantization, the Hamiltonian reads (assuming no dipole moment for the impurity $\downarrow$ atoms):

$$
\begin{aligned}
H= & \sum_{j} j a_{j}^{\dagger} a_{j}+\frac{g}{2} \sum_{(i k) \in \uparrow,(j l) \in \downarrow} V_{i j k l}^{\text {contact }} a_{i}^{\dagger} a_{j}^{\dagger} a_{k} a_{l} \\
& +\frac{u}{2} \sum_{(i j k l) \in \uparrow} V_{i j k l}^{\text {dipolar }} a_{i}^{\dagger} a_{j}^{\dagger} a_{k} a_{l},
\end{aligned}
$$

with annihilation and creation operators $a_{j}$ and $a_{j}^{\dagger}$, where the index $j$ refers to the $j$ th orbital, while the index for the spin has been suppressed. A constant energy term $N \hbar \omega / 2$, corresponding to the zero-point energy of the harmonic oscillator with $N$ particles, is neglected. With $\varphi_{j}(x)$ denoting the normalized orbitals, the interaction matrix elements are defined as follows:

and

$$
V_{i j k l}^{\text {contact }}=\int_{-\infty}^{\infty} d x \varphi_{i}(x) \varphi_{j}(x) \varphi_{k}(x) \varphi_{l}(x)
$$

$$
\begin{aligned}
V_{i j k l}^{\text {dipolar }}= & \int_{-\infty}^{\infty} d x d x^{\prime} U\left(x / \lambda-x^{\prime} / \lambda\right) \\
& \times \varphi_{i}(x) \varphi_{j}\left(x^{\prime}\right) \varphi_{k}(x) \varphi_{l}\left(x^{\prime}\right) .
\end{aligned}
$$

Both integrals can be solved by integrating out the center-ofmass coordinate $x+x^{\prime}$. While the remaining integral in the relative coordinate $x-x^{\prime}$ is trivial for the contact interaction, some care must be taken for the dipolar potential. We describe how to evaluate this integral in the appendix.

Experimentally, the most advanced platform to realize our system are ultracold atoms with strong magnetic dipole moments, such as chromium, erbium, and dysprosium. For instance, a fermionic gas of ${ }^{161}$ Dy has been cooled to quantum degeneracy in Ref. [33], with an outstandingly large dipole moment of ten Bohr magnetons in the atomic ground states, and without losses due to chemical reactions and inelastic collisions. Moreover, dysprosium has more than 140 metastable states, among which one also encounters unmagnetized states [30]. Thus, it is a good candidate for realizing a system of dipolar majority particles and impurities of equal mass without dipole moment. The dipolar interaction strength is maximally attractive for $\theta=0$, for which in the case of ${ }^{161}$ Dy we obtain $u /(\hbar \omega)=-0.016(\omega \mathrm{s})^{1 / 2}$. For $\theta=\pi / 2$, the interaction becomes maximally repulsive, with $u /(\hbar \omega)=$ $0.008(\omega \mathrm{s})^{1 / 2}$. With values of the trap frequency typically of the order of $\mathrm{kHz}$, we have $|u| /(\hbar \omega) \lesssim 1$. Larger values for $u$ can be achieved on other experimental platforms: Rydberg atoms 
have an induced electric dipole moment which scales like $n^{2}$, where $n$ is the principal quantum number. This allows for values of $|u|$ being some orders of magnitude larger than for magnetic atoms. In the context of Rydberg atoms [39-41], we can also realize the coexistence of majority particles with dipole moments and impurity particles without dipole moment: The majority atoms are given by the highly excited Rydberg atoms, while the impurity is an unexcited atom of the same species. Finally we note that very large permanent electric dipole moments are also present in heteronuclear molecules [37,38].

\section{TIME EVOLUTION AND THERMODYNAMICS}

In this section, we define different quantities which are useful for analyzing the dynamical properties of a quantum system. We are interested in scenarios where the system is initially prepared in some pure state $\left|\Psi_{\text {ini }}\right\rangle$ and then evolves in the Schrödinger picture under the time-independent Hamiltonian $H$. The time-evolved state is then given by

$$
|\Psi(t)\rangle=e^{-i H t}\left|\Psi_{\mathrm{ini}}\right\rangle=\sum_{\alpha} c_{\alpha} e^{-i E_{\alpha} t}|\alpha\rangle .
$$

Here, we have decomposed the initial state into eigenstates $|\alpha\rangle$ of $H, H|\alpha\rangle=E_{\alpha}|\alpha\rangle$. The coefficients $c_{\alpha}$ are given by $c_{\alpha}=\left\langle\alpha \mid \Psi_{\text {ini }}\right\rangle$.

Using exact diagonalization, we can determine the full energy spectrum of a small system and then straightforwardly calculate the evolution of an arbitrary state. The state vector itself, however, is not accessible experimentally. In practice, the system dynamics can be tracked by looking at the evolution of any observable. From the computational point of view, this requires evaluating the quantum average of operators $O:\langle O(t)\rangle=\langle\Psi(t)|O| \Psi(t)\rangle$. If all eigenstates $\alpha$ are nondegenerate, the temporal average of $\langle O(t)\rangle$, denoted by $\langle\langle O(t)\rangle\rangle_{t}$, will, for sufficiently long times, converge to a value described by the diagonal ensemble, $O_{\mathrm{DE}}$ :

$$
\begin{aligned}
\langle\langle O(t)\rangle\rangle_{t} & =\left\langle\sum_{\alpha, \beta} c_{\beta}^{*} c_{\alpha} e^{-i\left(E_{\alpha}-E_{\beta}\right) t}\langle\beta|O| \alpha\rangle\right\rangle_{t} \\
& \rightarrow \sum_{\alpha}\left|c_{\alpha}\right|^{2}\langle\alpha|O| \alpha\rangle \equiv O_{\mathrm{DE}}
\end{aligned}
$$

In the limit on the right-hand side, any off-diagonal contributions $\langle\alpha|O| \beta\rangle$ have been averaged away by the oscillatory factor. Whether and when not only the temporal average but also $\langle O(t)\rangle$ itself converge to $O_{\mathrm{DE}}$ is an interesting question of quantum thermodynamics [50-52].

In contrast to the diagonal ensemble, which may keep some memory of the initial state, thermal ensembles define the state of a system only by a few thermodynamic parameters which are fixed by the initial conditions. For example, if the canonical ensemble is applied, the state of the system is defined by the temperature $T$. On the other hand, to associate a temperature with the state of the system we evaluate the ensemble average $E_{\mathrm{th}}(T)$ of the energy:

$$
E_{\mathrm{th}}(T)=\frac{1}{Z} \operatorname{Tr}\left(H e^{-H / T}\right),
$$

where the Boltzmann constant has been set to 1 , and $Z$ denotes the partition function $Z=\operatorname{Tr} e^{-H / T}$. Comparison of the thermal energy $E_{\mathrm{th}}(T)$ with the system energy, $E=\left\langle\Psi_{\text {ini }}|H| \Psi_{\text {ini }}\right\rangle$, yields an effective temperature. With this, one is able to calculate thermal expectation values of any observables:

$$
O_{\mathrm{CE}}(T)=\frac{1}{Z} \operatorname{Tr}\left(O e^{-H / T}\right) .
$$

The observable which, in the present study, is used to track the system is the harmonic oscillator level of the impurity. Experimentally, this quantity can be determined by reducing the height of the trap. At a certain height, the impurity escapes the trap, and from this value, the oscillator level of the impurity can be determined. Formally, the corresponding operator reads $m_{\text {imp }}=\sum_{m} m\left|n_{m}\right\rangle_{\downarrow}\left\langle\left. n_{m}\right|_{\downarrow}\right.$. It acts only on the impurity (that is, the $\downarrow$ component), and counts the number of particles $n_{m}$ in each orbital $m$, weighted by the level number. We can associate $\left\langle m_{\mathrm{imp}}\right\rangle$ with the energy of the impurity.

\section{GROUND-STATE PROPERTIES}

Before studying the relaxation dynamics of the system in the next section, let us first discuss some static properties of the ground state. As has been demonstrated in Ref. [4], it is possible to measure the interaction energy of the impurity with high accuracy. The measurement is based on determining the resonance frequency for an internal transition of the impurity atom, which, in the presence of majority atoms, is shifted by the amount of the interaction energy. Increasing the number of majority atoms from one to five, it was shown that the interaction energy quickly approaches the theoretical predictions made for an impurity in a homogeneous Fermi sea [74]. Here, we will equip the majority atoms with a dipole moment; that is, the Fermi sea becomes interacting. As we show below, the interaction energy of the impurity provides information also about the strength of the dipolar interactions, although they are restricted to the majority atoms.

The system is described by the Hamiltonian $H$ of Eq. (3). Two control parameters, $u$ and $g$, allow us to adjust independently the interaction between the majority atoms and the interactions with the impurity. Using numerical diagonalization we have determined the total energy $E(u, g)$ for different dipolar and contact interaction strengths. We obtain the interaction energy of the impurity with the majority atoms as the difference $E(u, g)-E(u, 0)$. As shown in Fig. 1, repulsive interactions between the majority atoms $(u>0)$ lead to a reduced interaction energy of the impurity, while attractive dipolar interactions $(u<0)$ will increase the impurity's interaction energy.

An explanation for this behavior are density changes due to the dipolar interactions: Repulsive interactions reduce the local density of majority atoms near the trap center. Clearly, this also leads to decreased spatial overlap between majority atoms and impurity, since the impurity predominantly occupies the lowest oscillator level, and thus has an amplitude maximum at the center. As a consequence, the contact interaction energy between both species is reduced. Oppositely, attractive dipolar interactions lead to an increased density of majority atoms in the center of the trap and therefore enhance the contact interactions.

To quantify how strong the ground state is affected by the dipolar interactions, let us have a look onto overlaps between 


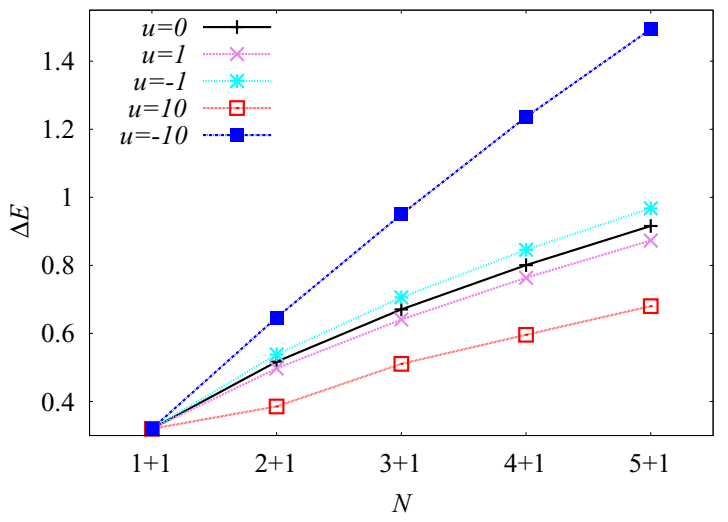

FIG. 1. (Color online) Interaction energy (in units $\hbar \omega$ ) of an impurity atom with several majority atoms as a function of the system size. The black line shows the behavior for a system of noninteracting majority atoms, $u=0$. Repulsive dipolar interactions between the majority atoms, $u>0$, increase the interaction energy, whereas attractive dipolar interactions decrease the interaction energy. The contact interaction strength $g$ between majority atoms and impurity has been set to $g=1$.

the ground state for different $u$ at $g=1$, for $3+1$ particles: The overlap between the ground state at $u=0$ and the ground state at $u=1$ is very large, $>0.99$. The same is true for the overlap between ground states at $u=0$ and $u=-1$. Even for strong repulsive interactions, $u=10$, the overlap with the ground state at $u=0$ remains larger than 0.82 , but it decreases to 0.34 in the case of strong attractive dipole-dipole interactions, $u=-10$. This demonstrates that significant modifications of ground-state properties can only be expected for strongly attractive dipolar interactions.

\section{DYNAMIC PROPERTIES: CASE STUDY}

We now investigate how the dipolar interactions between the majority atoms modify the properties of the system dynamics. Again, the impurity atom provides a tool for controlling and measuring the system. More concretely, we propose to study the time evolution of a system out of equilibrium by tracking the energy of the impurity. This is in analogy to recent studies of homogeneous quantum liquids in one dimension where the dynamics of an impurity has been determined using the Bethe ansatz [67], matrix product states [68], and Boltzmann kinetic theory [69-71]. Here, we consider the dynamics of an impurity in a harmonically trapped quantum gas by applying exact diagonalization.

Numerically, determining the time evolution is an extremely hard task, as knowledge of all eigenstates is required, while exact diagonalization algorithms work most efficiently if only a few eigenvectors are obtained. We therefore restrict the system size to $3+1$ atoms. Taking into account parity symmetry of the Hamiltonian, and restricting the single-particle basis to 22 (20) states, we have Hilbert space blocks of 16940 (11400) states. We have checked our results for convergence with respect to increases in the single-particle basis. Clearly, truncation errors are typically more pronounced for excited states than for the ground state, and time evolution exponentiates every error. Accordingly, in some cases, convergence can be achieved only on short time scales.

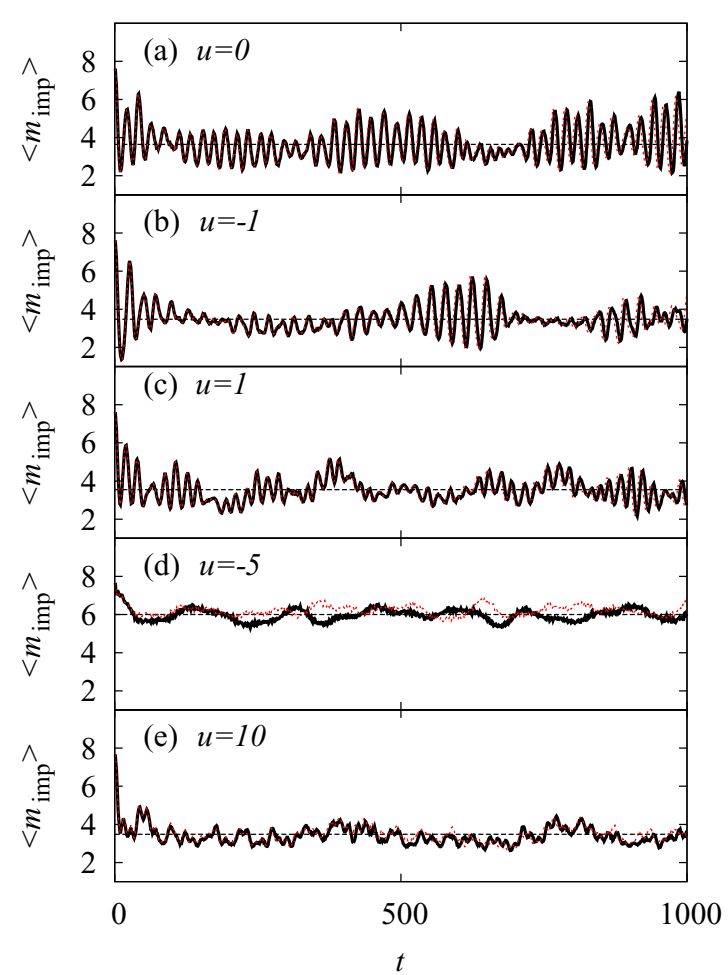

FIG. 2. (Color online) Time evolution tracked by the average level number $\langle m\rangle_{\text {imp }}$ of the impurity. The initial state is a Fock state with the three majority atoms in the three lowest levels, and the impurity occupying the 8 th excited level, $n_{\text {imp }}=8$. In all plots, the Hamiltonian $H$ contains a repulsive contact interaction of the majority atoms with the impurity $(g=1)$. The strength $u$ of the dipolar interactions between the majority atoms takes the value denoted in each plot. The horizontal dashed lines show the corresponding diagonal ensemble average. The red (gray) dotted line indicates whether the numerical treatment is converged: The black line shows the results taking into account 20 [panels (a)-(c)] or 22 [panels (d) and (e)] harmonic oscillator levels. The red (gray) dotted line shows the results for a smaller Hilbert space, taking into account 18 [panels (a)-(c)] or 20 [panels (d) and (e)] levels. The time $t$ is given in units $\omega^{-1}$.

We choose an easy-to-prepare initial state at relatively low energy. A convenient choice, taken for Fig. 2, is a Fock state with the three majority atoms populating the three lowest levels. In the absence of an impurity, this is the ground state of a system without dipolar interactions. The impurity is used to bring the system out of equilibrium. To have pronounced effects, the impurity shall occupy a level significantly above the equilibrium value. We have chosen $m_{\text {imp }}=8$ in Fig. 2. We then studied the time evolution of this initial state under the Hamiltonian $H$ of Eq. (3) for a contact interaction strength $g=1$, and for different values $u$ for the dipolar interactions between the majority atoms. Being a Fock state, the initial state is an (excited) eigenstate of the noninteracting Hamiltonian, $g=u=0$. This allows us to view the scenario also as an interaction quench, during which both contact and dipolar interactions are suddenly switched on.

Even without quantitative analysis, it is obvious from Fig. 2 that relaxation does not take place in the absence of dipolar interactions [Fig. 2(a)]. In this case, the system evolution is characterized by an oscillatory behavior for all 
TABLE I. Quantitative analysis of the data presented in Fig. 2.

\begin{tabular}{lccc}
\hline \hline & $\mu \pm \sigma$ & $S$ & $\left\langle V_{\mathrm{dd}}\right\rangle / \hbar \omega$ \\
\hline$g=1, u=0$ & $3.7 \pm 0.9$ & 2.7 & 0 \\
$g=1, u=-1$ & $3.5 \pm 0.7$ & 2.8 & -0.4 \\
$g=1, u=1$ & $3.6 \pm 0.5$ & 2.8 & 0.4 \\
$g=1, u=-5$ & $6.0 \pm 0.3$ & 2.4 & -2.1 \\
$g=1, u=10$ & $3.4 \pm 0.4$ & 4.1 & 4.1 \\
\hline \hline
\end{tabular}

times. In particular, even after a long relaxation time, the amplitude $\delta\left\langle m_{\text {imp }}\right\rangle$ of these oscillations still attains large values, $\delta\left\langle m_{\text {imp }}\right\rangle>1$. On average for long times $(350<t<$ $1000)$, the impurity level $\left\langle m_{\text {imp }}\right\rangle(t)$ oscillates around a mean $\mu=3.7$, close to the diagonal ensemble average of 3.6 , with a standard deviation $\sigma=0.9$, see Table I for a quantitative evaluation of Fig. 2.

One might argue that the observed regularity of the dynamics is due to the weakness of interactions, and therefore the proximity of the initial state to an eigenstate of the Hamiltonian $H$. As a quantitative measure for the amount of Hamiltonian eigenstates participating in the initial state, we define a kind of entropy

$$
S=-\sum_{\alpha}\left|c_{\alpha}\right|^{2} \ln \left|c_{\alpha}\right|^{2},
$$

with the $c_{\alpha}$ being the coefficients of a decomposition of the initial state in the eigenbasis of $H$; cf. Eq. (6). If $S=0$, the initial state is an eigenstate of $H$. If $S=\ln D, D$ eigenstates contribute with equal weights. Indeed, for the Hamiltonian of Fig. 2(a), the entropy of the initial state takes only a moderate value, $S=2.7$. Also, the average interaction energy $\langle V\rangle=0.2$ is small compared to the total energy, $\langle H\rangle=11.2$. However, even for an increased contact interaction $g=10$ (and $u=0)$, the dynamics remains regular (see Fig. 3), although now entropy and interaction energy are significantly larger, $S=4.1$ and $\langle V\rangle=2.4$. This suggests that it is not the weakness of the quench which led to the regular dynamics in Fig. 2(a). We note that for $g=10$, the time evolution is quantitatively not converged for large times $t \gtrsim 100$. Qualitatively, however, the evolution remains regular on all time scales, independently from the number of single-particle states taken into account.

Next, we consider the case of weak dipolar interactions between the majority atoms, in addition to weak contact interactions with the impurity; see Figs. 2(b) and 2(c). The

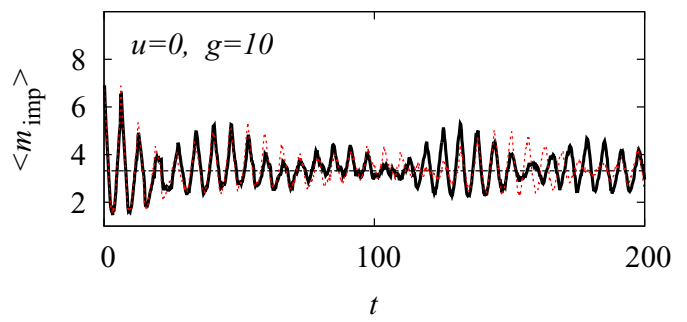

FIG. 3. (Color online) Time evolution as in Fig. 2, but with strong contact interactions, $g=10$, and no dipolar interactions, $u=10$. The black line (red [gray] dotted line) shows the results taking into account 22 (20) harmonic oscillator levels. The horizontal dashed line indicates the diagonal ensemble average. The time $t$ is given in units $\omega^{-1}$. oscillation amplitudes are reduced compared to the case without dipolar interactions, with a long-time mean $\mu=3.5 \pm 0.7$ for attractive interactions $(u=-1)$, and $\mu=3.6 \pm 0.5$ for repulsive interactions $(u=1)$, in agreement with the diagonal ensemble average (3.5 in both cases). However, in particular for the attractive system, periods of strong oscillations occur repeatedly even for long relaxation times, keeping the system away from a steady state. In both the attractive and the repulsive case, the entropy of the initial state is $S=2.8$. Additionally to the contact interaction energy, $\left\langle V_{\mathrm{c}}\right\rangle=0.2$, the total interaction energy now contains also a dipolar contribution: $\langle V\rangle=\left\langle V_{\mathrm{dd}}\right\rangle+\left\langle V_{\mathrm{c}}\right\rangle$. The dipolar contribution takes the values $\left\langle V_{\mathrm{dd}}\right\rangle= \pm 0.4$ for $u=\mp 1$.

In Figs. 2(d) and 2(e), we turn our attention to strong dipolar interactions, $u=-5$ and $u=10$. Since attractive interactions have a stronger tendency to populate higher oscillator levels, we are not able to achieve numerical results which remain convergent for long times. As seen in Fig. 2(d), the time evolution for $u=-5$ is quantitatively not converged for $t \gtrsim 50$. This means that only the initial decay of $\left\langle n_{\text {imp }}\right\rangle(t)$ is captured accurately. However, for larger times the evolution consists only of small-valued fluctuations around a mean value corresponding to the diagonal ensemble average $(\mu=6.0 \pm 0.3$ for a basis with 22 states, and $\mu=6.2 \pm 0.3$ for a basis with 20 states). This suggests that the system quickly reaches a steady state, despite the remarkably small entropy of the initial state, $S=2.4$. The interaction energy is $\left\langle V_{\mathrm{dd}}\right\rangle=-2.1$.

For strongly repulsive dipolar interactions, shown in Fig. 2(e), we are able to obtain fairly well-converged results even for long relaxation times. The system quickly evolves towards the average of the diagonal ensemble (3.4), around which it fluctuates in an erratic manner with a standard deviation $\sigma=0.4$. This means that the deviations from the average are only slightly smaller than for weak repulsive interaction, Fig. 2(c). Instead, it is the absence of oscillatory behavior which make the strongly interacting system appear significantly more relaxed than the weakly interacting one. Both average dipolar interaction energy and entropy of the initial state are large: $\left\langle V_{\mathrm{dd}}\right\rangle=4.1$ and $S=4.4$.

Finally, we may ask whether this relaxed state is also thermalized. In the canonical ensemble, energy $E$ is associated with temperature $T$ according to Eq. (8). This leads to a gauge curve $E(T)$ plotted in Fig. 4(a) for $g=1, u=0$, and 10. From

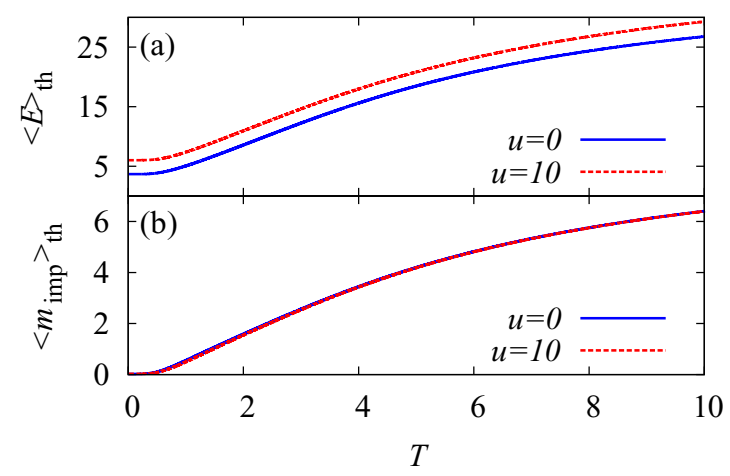

FIG. 4. (Color online) Canonical ensemble averages of (a) energy $E_{\text {th }}$ (in units $\hbar \omega$ ) and (b) impurity oscillator level $\left\langle m_{\text {imp }}\right\rangle$, as a function of temperature $T$ (in units $\hbar \omega / k_{\mathrm{B}}$ ). 
this curve, we infer an effective temperature $T=3.2$ for the system with strong dipolar interactions. On the other hand, using the canonical ensemble Eq. (9), we can also evaluate the thermal average of $n_{\text {imp }}$ for any given temperature. The question is whether $n_{\text {imp }}$ relaxes to the value which corresponds to the effective temperature defined via the energy. The temperature dependence of $n_{\text {imp }}$, which has basically no dependence on $u$, is plotted in Fig. 4(b). For $T=3.2$, we find the thermal average $\left\langle n_{\text {imp }}\right\rangle_{\text {th }} \approx 2.8$, which is significantly below the temporal average in Fig. 2(e), around 3.5. This comparison shows that the system, although it relaxes, does not thermalize.

\section{DYNAMIC PROPERTIES: DISCUSSION}

Let us summarize the main findings of the case studies presented in the previous section:

(1) Relaxation towards the diagonal ensemble average occurs in the presence of sufficiently strong dipolar interactions between the majority atoms.

(2) The system dynamics remains oscillatory for a system with purely contact interactions. This seems not to be related to a smaller interaction energy, nor to a smaller participation entropy.

(3) The system, even when it relaxes, does not thermalize.

Let us now try to understand the differences in the relaxation behavior from a more general point of view. A concept which will prove useful is the distinction between chaotic and regular dynamics: While regular dynamics preserves the coherences of the initial state, chaotic evolution should quickly lead to relaxation. Then, the different behavior should be reflected by the energy spectra. In quantum mechanics, chaotic behavior is characterized by level repulsion. For a system with time-reversal symmetry, the Hamiltonian matrix is real symmetric, and random matrix theory, cf. Ref. [75], tells us that the level spacing should follow a Wigner distribution function, $P_{\text {Wigner }}(s)=\frac{\pi}{2} s e^{-s^{2} \pi / 4}$. Clearly, the maximum of this distribution is located at finite spacings $s=\sqrt{2 / \pi}$. On the other hand, such level repulsion is not expected for a system with regular dynamics. In this case, conserved quantities will allow energy levels to cross; that is, correlations between the levels are absent. This may lead to a Poissonian level spacing distribution, $P_{\text {Poisson }}(s)=e^{-s}$, with a maximum for $s=0$.

Before analyzing the level spacing distribution, it is necessary to unfold the energy spectra. Naturally, level spacings will be larger in spectral regions with a low density of states than in dense regions, but this obviously has nothing to do with correlations between energy levels. By unfolding the energy spectrum, we guarantee an overall homogeneous density of states, such that level correlations become the only source for variations in the level spacing. To that aim, we follow the unfolding procedure described in Ref. [75]. The first step is to smooth the discrete density of states via a convolution with a Gaussian:

$$
\rho_{\text {smooth }}(E)=\frac{1}{N} \sum_{i=1}^{N} \frac{1}{\Delta \sqrt{\pi}} e^{-\left(E-E_{i}\right)^{2} / \Delta^{2}} .
$$

We have chosen the width $\Delta$ of the Gaussian to be given by five times the mean level spacing. The smoothened density of states also leads to a smooth staircase function $\Sigma_{\text {smooth }}(E)$, that

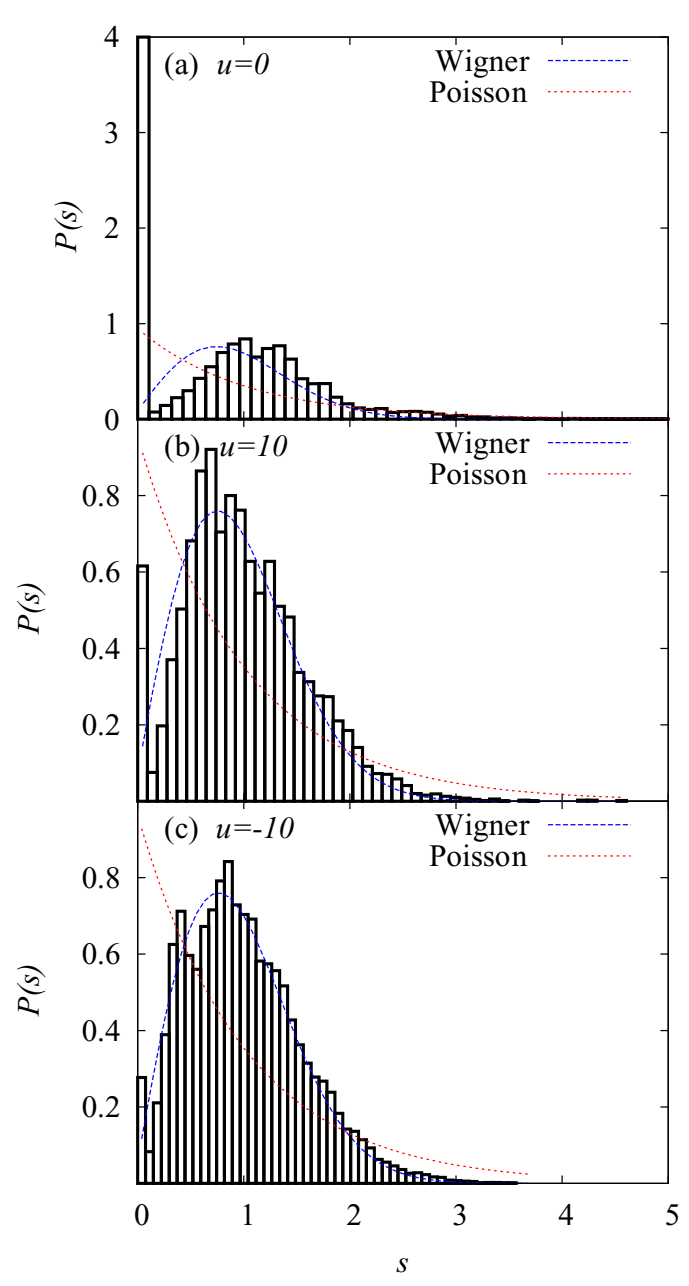

FIG. 5. (Color online) Unfolded level spacing in energy spectra of $H$ with $g=1$ and for different $u$. For normalizing the distribution, we did not take into account levels at $s=0$.

is, a smooth cumulated density of states:

$$
\Sigma_{\text {smooth }}(E)=\int_{-\infty}^{E} d E^{\prime} \rho_{\text {smooth }}\left(E^{\prime}\right) .
$$

The unfolded energy levels $e_{i}$ are then associated with $e_{i}=N \Sigma_{\text {smooth }}\left(E_{i}\right)$, and the unfolded level spacings are $s_{i}=e_{i}-e_{i-1}$.

We have evaluated the unfolded level spacing distribution for the energy spectrum of $H$ with $g=1$ and $u=0,10,-10$, for $3+1$ atoms, taking into account 20 harmonic oscillator levels. In the presence of dipolar interactions, as seen in Figs. 5(b) and 5(c), the level spacing is well described by the Wigner distribution, except for a somewhat increased number of levels near $s=0$ in the case of repulsive interactions; see Fig. 5(b). Nevertheless, the distributions clearly indicate level repulsion, and thus chaotic dynamics. This is in agreement with the relaxation properties discussed in the previous section. More complicated to interpret is the distribution in the absence of dipolar interactions, Fig. 5(a). Neither the Wigner nor the Poisson distribution capture the level spacing distribution. On the other hand, an eye-catching property of the distribution is the exorbitantly large number of levels near $s \approx 0$, with around 4400 of 11399 spacings peaked around zero. This number is 
not compatible even with a Poisson distribution, for which around $10 \%$ of the levels should be located within $s<0.1$ [78]. The distribution of the remaining levels is neither described by a Poisson nor by a Wigner function, even if we exclude the $s=0$ levels from the normalization (which leads to a normalized distribution of the remaining levels). The Wigner distribution catches well only the behavior at intermediate spacing, $1 \lesssim s \lesssim 2$, whereas the tail of the distribution has Poissonian shape. It might be that the huge number of degenerate levels spoils our unfolding procedure and/or that a mixed phase space, consisting of both chaotic and regular regimes, leads to such unconventional distribution function. As a conclusion, the dominant feature of the distribution is the abundantly many degeneracies, and therefore we may take the observed level spacing as an indicator for a regular system dynamics.

An observation which might, at least partly, explain the large number of nearby levels is the quasi-integrability of the model without dipolar interactions: First, there is an exact solution for $1+1$ particles in a harmonic trap [76], and a quasiexact ansatz for $N+1$ particle [16]. Second, in the homogeneous case with $N+1$ particles, the system is integrable via Bethe ansatz [74,77]. Finally, although no exact ground-state solution is known for the trapped system with $N+1$ fermions, exact wave functions are easily obtained for several excited states due to a fermionization of the problem: In a fermionized picture, we view the two-component Fermi system with contact interactions between the components as a noninteracting one-component Fermi system. Since the spatial wave functions of the noninteracting Fermi system, corresponding to Slater determinants, incorporate the Pauli principle, these wave functions fully suppress contact interactions, and therefore they are also eigenfunctions of the two-component system. While the energy of these solutions is independent of $g$, and the solutions exist for any $g$, one solution becomes (quasi)degenerate with the ground state for $g \rightarrow \infty$. For any $g$, the Slater states play an important dynamical role, as the degeneracies in the energy spectra can be traced back to them: The energy of a Slater state corresponds to the single-particle energies of the $N+1$ different harmonic oscillator levels which are filled. At larger energies, there is a rapidly growing number of ways how $N+1$ particles can be distributed, leading to an increasing number of fermionized solutions. In the harmonic trap potential, with equidistant energy levels, many choices of single-particle levels add up to the same total energy, and accordingly we get a huge number of degenerate many-body states. In this context, it is interesting to note that for strongly repulsive contact interactions, the energies of all eigenstates approach the values given by the Slater states $[6,16]$. In that case, energy levels are either degenerate or separated by the integer spacing $\hbar \omega$, which apparently would lead to a very atypical bimodal level spacing distribution.

Let us finally study why the system, even for cases in which it equilibrates, lacks thermalization. A hypothesis explaining thermalization of an isolated quantum system is the eigenstate thermalization hypothesis $[45,46]$. It assumes that, for any eigenstate, the quantum average of an operator and the corresponding eigenenergy are correlated; that is, all eigenstates in the vicinity of some energy $E$ have the same

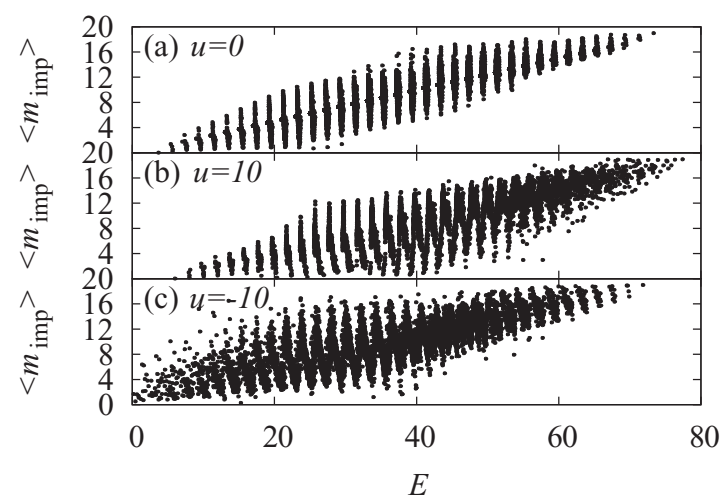

FIG. 6. We plot the average occupation $m_{\text {imp }}$ vs the energy (in units $\hbar \omega$ ) for all eigenstates of $H$, with $g=1$ and for different $u$.

expectation values with respect to relevant observables $O$. Moreover, these values are assumed to be thermalized; that is, they coincide with the thermodynamic average. We test the eigenstate thermalization hypothesis by plotting the average occupation $\left\langle m_{\mathrm{imp}}\right\rangle$ versus the energy for all eigenstates in Fig. 6. In both cases, with or without dipolar interactions, the values of $\left\langle m_{\mathrm{imp}}\right\rangle$ are spread over a broad range for almost any energy in the spectrum. Accordingly, the eigenstate thermalization hypothesis does not hold, and fixing the energy in a microcanonical ensemble will not fix the expectation value of $m_{\mathrm{imp}}$. This explains why thermalization does not take place.

\section{SUMMARY AND OUTLOOK}

In summary, we have suggested probing the dynamics of a Fermi system trapped in one dimension by exciting an impurity and tracking the oscillator level of the impurity. We have studied a system with dipolar interactions and have contrasted its dynamics to the dynamics of a system with purely contact interactions. While the latter shows quantum collapse and revival effects even after long relaxation times, the system with sufficiently strong dipolar interactions relaxes quickly to its diagonal ensemble average. We relate this finding to the level spacing distribution, which indicates chaotic behavior in the presence of dipolar interactions. In the case without dipolar interactions, a huge number of degenerate levels characterizes the level spacing distribution.

Even despite the small system size of only four fermions, we encounter cases where the time evolution cannot be computed faithfully on long time scales. This demonstrates the need for better computational techniques, and/or alternative approaches such as quantum simulations. Our work suggests exploring dynamical aspects in the experimental setting of Ref. [4]. In view of the recent experimental progress with dipolar atoms [35,36,41] and molecules [38], scenarios as the one studied in this paper are becoming experimentally feasible.

In our study, we have assumed majority particles and impurity to be of equal mass, but with different dipole moments. Moreover, we have assumed a harmonic trap potential with an anisotropy parameter $\lambda=1 / 3$. For the effective dipole-dipole interaction in one dimension, the single-mode approximation was used, restricting the transverse modes to their groundstate level. While all these details can be justified for some 
realizations, we should bare in mind that any modification might change the system behavior quantitatively or even qualitatively. For instance, in a slightly modified scenario, majority atoms and impurities could be two different species with unequal masses: This would lead to a smaller momentum transfer in a scattering event between majority atom and impurity, and one therefore expects increased relaxation times. Another possible modification is related to the anisotropy of the traps: Smaller values of $\lambda$ would change the effective potential towards a $\delta$-like shape, decreasing the effect of the dipolar interactions. For larger values of $\lambda$, the single-mode approximation might fail, and, within a multimode description, the effective potential acquires both repulsive and attractive parts; cf. Ref. [44]. Intriguing effects could also be brought in by trap anharmonicities: As argued in Sec. VI, a huge number of degeneracies is present in the spectrum without dipolar interactions, but the number of degeneracies is expected to be much smaller in an unharmonic trap. As a consequence, anharmonicities might enhance chaotic behavior even in the absence of dipolar interactions.

\section{ACKNOWLEDGMENTS}

I am grateful to Maciej Lewenstein for reading and commenting on the manuscript and for discussions. I also thank Miguel Angel Garcia-March, Tomek Grining, Pietro Massignan, and Michal Tomza for discussions. Financial support from EU OSYRIS (ERC-2013-AdG Grant No. 339106), SIQS (FP7-ICT-2011-9 No. 600645), QUIC
(H2020-FETPROACT-2014 No. 641122), and EQuaM (FP7/2007-2013 Grant No. 323714); Spanish Ministry Grant FOQUS (FIS2013-46768-P); and Fundació Cellex is acknowledged.

\section{APPENDIX: DIPOLE MATRIX ELEMENTS}

We need to evaluate matrix elements $V_{i j k l}^{\text {dipolar }}=$ $\int_{-\infty}^{\infty} d x d x^{\prime} U\left(x / \lambda-x^{\prime} / \lambda\right) \varphi_{i}(x) \varphi_{j}\left(x^{\prime}\right) \varphi_{k}(x) \varphi_{l}\left(x^{\prime}\right)$ for a twobody potential

$$
U(x)=-2|x|+\sqrt{2 \pi}\left(1+x^{2}\right) e^{x^{2} / 2} \operatorname{erfc}(x / \sqrt{2}) .
$$

The orbitals $\varphi_{i}(x)$ are the usual harmonic oscillator levels

$$
\varphi_{j}(x)=\sqrt{\frac{1}{\pi^{1 / 2} 2^{j} j !}} e^{-x^{2} / 2} H_{n}(x) \equiv N_{j} e^{-x^{2} / 2} H_{n}(x),
$$

where $H_{n}(x)$ are the Hermite polynomials, and $N_{j}$ denotes the normalization constant of the wave function. To evaluate the integral, we replace the coordinates $x$ and $x^{\prime}$ by relative coordinates $r=x-x^{\prime}$ and center-of-mass coordinates $R=$ $x+x^{\prime}$. The integral over the center-of-mass part, $I_{R}^{(i)}$, reduces to

$$
I_{R}^{(i)} \equiv \int_{-\infty}^{\infty} R^{i-1} e^{-R^{2} / 2} d R=2^{i / 2-1}\left[1+(-1)^{i+1}\right] \Gamma\left(\frac{i}{2}\right),
$$

with $i$ some positive integer. Also the integral over the relative coordinate, $I_{r}^{(i)}$, turns out to have a compact solution:

$$
\begin{aligned}
I_{r}^{(i)} \equiv & \int_{-\infty}^{\infty} r^{i-1} e^{-r^{2} / 2}\left[-2\left|\frac{r}{\lambda}\right|+\sqrt{2 \pi}\left(1+\frac{r^{2}}{\lambda^{2}}\right) e^{(r / \lambda)^{2} / 2} \operatorname{erfc}\left(\left|\frac{r}{\sqrt{2} \lambda}\right|\right)\right] d r \\
= & \frac{1}{\lambda} 2^{-\frac{1}{2}-\frac{i}{2}}\left[-1+(-1)^{i}\right]\left[2^{i+1} \Gamma\left(\frac{i+1}{2}\right)-2 \sqrt{\pi} \lambda^{i+1} \Gamma(i)_{2} \tilde{F}_{1}\left(\frac{i}{2}, \frac{i+1}{2}, \frac{i+2}{2} ; 1-\lambda^{2}\right)\right. \\
& \left.-\sqrt{\pi} \lambda^{i+1} \Gamma(i+2)_{2} \tilde{F}_{1}\left(\frac{i}{2}, \frac{i+1}{2}, \frac{i+2}{2} ; 1-\lambda^{2}\right)\right],
\end{aligned}
$$

where ${ }_{2} \tilde{F}_{1}(a, b, c ; z)$ is the regularized hypergeometrical function.

Any matrix element $V_{i j k l}^{\text {dipolar }}$ can be decomposed into a sum over products of these two integrals $I_{R}^{(i)}$ and $I_{r}^{(i)}$, with prefactors stemming from the Hermite polynomials and the normalization factors $N_{j}$ of the orbitals. However, care must be taken when numerically evaluating the matrix element in high orbitals. Since the numeric values of the integrals become large, while the normalization factors become small, it is crucial to keep symbolic expressions as long as possible to avoid numeric errors. A piece of MATHEMATICA code which performs the decomposition and evaluates the matrix element $V_{n 1 n 2 n 3 n 4}^{\text {dipolar }}$ reads

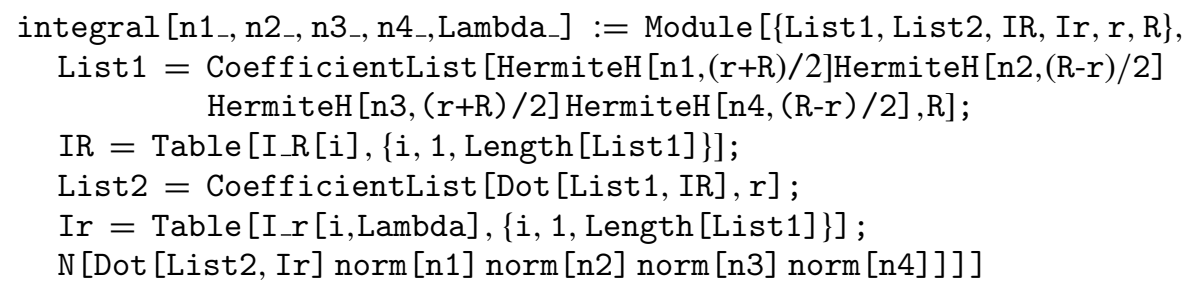

where $I_{-} R[i]$ is given by $I_{R}(i), I_{-} r[i, L a m b d a]$ is given by $I_{r}(i)$ for some choice of $\lambda$, and norm[n1] is the normalization factor $N_{n 1}$.

[1] M. Lewenstein, A. Sanpera, and V. Ahufinger, Ultracold Atoms in Optical Lattices: Simulating Quantum Many-Body Systems (Oxford University Press, Oxford, 2012).

[2] F. Serwane et al., Science 332, 336 (2011).
[3] G. Zürn, F. Serwane, T. Lompe, A. N. Wenz, M. G. Ries, J. E. Bohn, and S. Jochim, Phys. Rev. Lett. 108, 075303 (2012).

[4] A. N. Wenz et al., Science 342, 457 (2013).

[5] I. Brouzos and P. Schmelcher, Phys. Rev. A 87, 023605 (2013). 
[6] T. Sowiński, T. Grass, O. Dutta, and M. Lewenstein, Phys. Rev. A 88, 033607 (2013).

[7] G. E. Astrakharchik and I. Brouzos, Phys. Rev. A 88, 021602 (2013).

[8] S. E. Gharashi and D. Blume, Phys. Rev. Lett. 111, 045302 (2013).

[9] E. J. Lindgren et al., New J. Phys. 16, 063003 (2014).

[10] X. Cui and T.-L. Ho, Phys. Rev. A 89, 023611 (2014).

[11] S. E. Gharashi, X. Y. Yin, and D. Blume, Phys. Rev. A 89, 023603 (2014).

[12] A. G. Volosniev et al., Few-Body Syst. 55, 839 (2014).

[13] A. G. Volosniev et al., Nat. Commun. 5, 5300 (2014).

[14] N. P. Mehta, Phys. Rev. A 89, 052706 (2014).

[15] S. E. Gharashi, X. Y. Yin, Y. Yan, and D. Blume, Phys. Rev. A 91, 013620 (2015).

[16] J. Levinsen, P. Massignan, G. M. Bruun, and M. M. Parish, Sci. Adv. 1, e1500197 (2015).

[17] T. Grining et al., arXiv:1507.03174.

[18] F. Deuretzbacher, D. Becker, J. Bjerlin, S. M. Reimann, and L. Santos, Phys. Rev. A 90, 013611 (2014).

[19] A. G. Volosniev et al., New J. Phys. 15, 043046 (2013).

[20] N. T. Zinner et al., Europhys. Lett. 107, 60003 (2014).

[21] M. A. García-March, B. Juliá-Díaz, G. E. Astrakharchik, J. Boronat, and A. Polls, Phys. Rev. A 90, 063605 (2014).

[22] M. A. García-March et al., New J. Phys. 16, 103004 (2014).

[23] S. Campbell, M. A. García-March, T. Fogarty, and T. Busch, Phys. Rev. A 90, 013617 (2014).

[24] A. S. Dehkharghani, A. G. Volosniev, and N. T. Zinner, arXiv:1503.03725.

[25] P. Massignan, J. Levinsen, and M. M. Parish, arXiv:1507.02814.

[26] L. Santos, G. V. Shlyapnikov, P. Zoller, and M. Lewenstein, Phys. Rev. Lett. 85, 1791 (2000).

[27] L. Santos, G. V. Shlyapnikov, and M. Lewenstein, Phys. Rev. Lett. 90, 250403 (2003).

[28] K. Góral, L. Santos, and M. Lewenstein, Phys. Rev. Lett. 88, 170406 (2002).

[29] T. Lahaye et al., Nature (London) 448, 672 (2007).

[30] S. H. Youn, M. Lu, U. Ray, and B. L. Lev, Phys. Rev. A 82, 043425 (2010).

[31] A. R. P. Lima and A. Pelster, Phys. Rev. A 81, 021606 (2010).

[32] T. Graß, M. A. Baranov, and M. Lewenstein, Phys. Rev. A 84, 043605 (2011).

[33] M. Lu, N. Q. Burdick, and B. L. Lev, Phys. Rev. Lett. 108, 215301 (2012).

[34] M. A. Baranov, M. Dalmonte, G. Pupillo, and P. Zoller, Chem. Rev. 112, 5012 (2012).

[35] K. Aikawa, A. Frisch, M. Mark, S. Baier, R. Grimm, and F. Ferlaino, Phys. Rev. Lett. 112, 010404 (2014).

[36] A. de Paz et al., arXiv:1507.04273.

[37] K.-K. Ni et al., Science 322, 231 (2008).

[38] B. Yan et al., Nature (London) 501, 521 (2013).

[39] H. Weimer, R. Löw, T. Pfau, and H. P. Büchler, Phys. Rev. Lett. 101, 250601 (2008).

[40] J. Nipper, J. B. Balewski, A. T. Krupp, B. Butscher, R. Low, and T. Pfau, Phys. Rev. Lett. 108, 113001 (2012).

[41] D. Barredo, H. Labuhn, S. Ravets, T. Lahaye, A. Browaeys, and C. S. Adams, Phys. Rev. Lett. 114, 113002 (2015).

[42] S. Sinha and L. Santos, Phys. Rev. Lett. 99, 140406 (2007).

[43] F. Deuretzbacher, J. C. Cremon, and S. M. Reimann, Phys. Rev. A 81, 063616 (2010).
[44] L. Guan, X. Cui, R. Qi, and H. Zhai, Phys. Rev. A 89, 023604 (2014).

[45] J. M. Deutsch, Phys. Rev. A 43, 2046 (1991).

[46] M. Srednicki, Phys. Rev. E 50, 888 (1994).

[47] S. Hofferberth et al., Nature (London) 449, 324 (2007).

[48] T. Barthel and U. Schollwöck, Phys. Rev. Lett. 100, 100601 (2008).

[49] M. Cramer, C. M. Dawson, J. Eisert, and T. J. Osborne, Phys. Rev. Lett. 100, 030602 (2008).

[50] M. Rigol, V. Dunjko, and M. Olshanii, Nature (London) 452 , 854 (2008)

[51] M. Rigol, Phys. Rev. A 80, 053607 (2009).

[52] M. Rigol and M. Srednicki, Phys. Rev. Lett. 108, 110601 (2012).

[53] E. Khatami, G. Pupillo, M. Srednicki, and M. Rigol, Phys. Rev. Lett. 111, 050403 (2013).

[54] R. Steinigeweg, A. Khodja, H. Niemeyer, C. Gogolin, and J. Gemmer, Phys. Rev. Lett. 112, 130403 (2014).

[55] W. Beugeling, R. Moessner, and M. Haque, Phys. Rev. E 89, 042112 (2014).

[56] H. Kim, T. N. Ikeda, and D. A. Huse, Phys. Rev. E 90, 052105 (2014).

[57] A. Polkovnikov, K. Sengupta, A. Silva, and M. Vengalattore, Rev. Mod. Phys. 83, 863 (2011).

[58] J. Eisert, M. Friesdorf, and C. Gogolin, Nat. Phys. 11, 124 (2015).

[59] E. H. Lieb and D. W. Robinson, Commun. Math. Phys. 28, 251 (1972).

[60] M. B. Hastings and T. Koma, Commun. Math. Phys. 265, 781 (2006).

[61] P. Hauke and L. Tagliacozzo, Phys. Rev. Lett. 111, 207202 (2013).

[62] G. Carleo, F. Becca, L. Sanchez-Palencia, S. Sorella, and M. Fabrizio, Phys. Rev. A 89, 031602(R) (2014).

[63] T. Graß and M. Lewenstein, EPJ Quantum Technol. 1, 8 (2014).

[64] P. Jurcevic et al., Nature (London) 511, 202 (2014).

[65] P. Richerme et al., Nature (London) 511, 198 (2014).

[66] L. Cevolani, G. Carleo, and L. Sanchez-Palencia, arXiv: 1503.01786 .

[67] C. J. M. Mathy, M. B. Zvonarev, and E. Demler, Nat. Phys. 8, 881 (2012)

[68] M. Knap, C. J. M. Mathy, M. Ganahl, M. B. Zvonarev, and E. Demler, Phys. Rev. Lett. 112, 015302 (2014).

[69] E. Burovski, V. Cheianov, O. Gamayun, and O. Lychkovskiy, Phys. Rev. A 89, 041601 (2014).

[70] O. Gamayun, O. Lychkovskiy, and V. Cheianov, Phys. Rev. E 90, 032132 (2014).

[71] O. Gamayun, Phys. Rev. A 89, 063627 (2014).

[72] O. Lychkovskiy, Phys. Rev. A 89, 033619 (2014).

[73] O. Gamayun, A. G. Pronko, and M. B. Zvonarev, Nucl. Phys. B 892, 83 (2015).

[74] J. B. McGuire, J. Math. Phys. 6, 432 (1965).

[75] F. Haake, Quantum Signatures of Chaos (Springer, Berlin, 2001).

[76] T. Busch, B.-G. Englert, K. Rzaewski, and M. Wilkens, Found. Phys. 28, 549 (1998).

[77] X.-W. Guan, M. T. Batchelor, and C. Lee, Rev. Mod. Phys. 85, 1633 (2013).

[78] In Fig. 5(a), only levels at nonzero $s$ have been taken into account for normalizing the distribution. 